\title{
Parkinson's Disease, \\ Tourette Syndrome, and the \\ Changing Nature of Depression: \\ The Dog Days of Summer
}

By Eric Hollander, MD

Since the development of selective serotonin reuptake inhibitors there has been a widening of the definition of depression and a decrease in the placebo-drug difference in controlled studies. In the early 1960 s, $~ 33 \%$ of depressed patients improved with placebo and $66 \%$ with active compounds and current controlled studies suggest that the situation has certainly not improved. The Sequenced Treatment Alternatives to Relieve Depression Study found that response rates to new compounds after the failure of the first antidepressant are low. The monoamine hypothesis of depression was formulated in the mid 1960 s based on the antidepressant efficacy of the monoamine reuptake inhibitors, monoamine oxidase inhibitors and the depressogenic effects of reserpine as a monoamine depleter. However, no monoamine-related finding has been found that is diagnostic for depression. A second major hypothesis regarding depression has been the stress cortisol hypothesis. However, blood cortisol levels are not diagnostic of depression. Every psychiatric clinician is convinced that there are patients for whom antidepressants have made the difference between life and death. However, physicians may generalize unjustifiably based on single dramatic cases to a much larger diagnostic group. Perhaps there are many causes of different types of human sadness, and perhaps only some of these involve mechanisms related to monoamines. Thus, Robert $\mathrm{H}$. Belmaker, $M D$, suggests that perhaps only some kinds of depression are responsive to monoamine affecting antidepressants.

While Parkinson's disease is a neurodegenerative disorder characterized by bradykinesia, rigidity, postural instability and resting tremor, increasingly, it has also been associated with a broad spectrum of behavioral symptoms, such as sleep disorders, cognitive impairment, psychosis, depression, anxiety, and apathy. In addition, some Parkinson's disease patients develop compulsive and impulsive behaviors while receiving dopamine-replacement therapy, including: medication hoarding, pathological gambling, binge eating, hyperlibidinous behavior, compulsive shopping and punding. These behaviors may result in substantial psychosocial impairment for patients and therapeutic challenges for clinicians. Joseph M. Ferrara, MD, and Mark Stacy, $M D$, review the anatomic substrates, behavioral spectrum, associated factors and potential treatments for dopamine-replacement therapy-related compulsions in Parkinson's disease. These compulsive and impulsive side effects in response to dopamine agonist treatment have important implications for the understanding of the underlying conceptualization, neurobiology, and func-

Dr. Hollander is the editor of this journal, Esther and Joseph Klingenstein Professor and Chairman of Psychiatry at the Mount Sinai School of Medicine, and director of the Seaver and New York Autism Center of Excellence in New York City. 
tional neurocircuitry of impulse-control disorders and behavioral addictions.

Kevin M. Nasky, DO, and colleagues describes a case study of a female diagnosed with anti- $N$ methyl-D-aspartate (NMDA) receptor encephalitis without an identified tumor who presented with floridly psychotic symptoms following a 2-week prodromal phase with new-onset headaches and presyncopal episodes. While hospitalized, the patient had seizures, autonomic dysfunction, involuntary movements, and a decline in mental status. A subsequent assay was positive for antiNMDA receptor antibodies. In contrast to most reported cases, an initial trial with corticosteroids was therapeutically unsuccessful. Subsequent treatment with intravenous immunoglobulins, however, resulted in a prompt, robust clinical response and enabled the patient to be rapidly discharged from the hospital, with minimal neuropsychiatric sequelae. Identifying such a rare cause of such a complicated neuropsychiatric presentation, can, and did, have profound treatment implications. Sometimes, you need to look for zebras when you hear unusual hoofbeats.

Phenomenological, family, genetic, and treatment studies have suggested that tic related OCD could be different from non-tic-related OCD. T.S. Jaisoorya, MD, MRCPsych, and colleagues investigated the differences in obsessive-compulsive disorder (OCD) with and without tics, with respect to sociodemographics, symptom profile, and comorbidity, including obsessive- compulsive spectrum disorders. Subjects with OCD with tics tended to be males, and had an earlier onset of illness. They had more symmetry/ aggressive and religious obsessions, and cleaning, ordering/arranging, hoarding, and repeating compulsions and were associated with trichotillomania and hypochondraisis. An early age of onset, male gender, aggressive obsessions, cleaning compulsions, and trichotillomania were significantly associated with tic-related OCD. It is clear that tics contribute to the heterogeneity of $O C D$, and may represent a unique subtype or dimension within OCD.

This month's CNS Spectrums describes medication-related side effects (compulsivity and impulsivity) that can have important implication's for understanding symptom classification and the neurobiology of disease (behavioral addictions); assesses subtypes of illness (tic-related OCD) with distinct presentations; highlights changes in our understanding of illnesses such as depression over time (drug-nonresponsive sadness), and describes unusual cases with NMDA receptor encephalitis with highly specific treatment response (immunomodulatory treatment). CNS

\section{ERRATUM}

The "Clinical Updates in Neuropsychiatry" column in the May 2008 issue stated that the starting dosage of desvenlafaxine is $100 \mathrm{mg} /$ day. The correct dosage is $50 \mathrm{mg} /$ day. We regret the error. 